\title{
Cuatro nuevas especies del grupo Drosophila onychophora (Diptera, Drosophilidae) en el Parque Arqueológico Rumipamba, Pichincha, Ecuador
}

\author{
María Luna Figuero, Renata León, Violeta Rafael \& Diego Céspedes
}

Laboratorio de Genética Evolutiva, Escuela de Ciencias Biológicas, Pontificia Universidad Católica del Ecuador, 17-01-2184, Quito, Ecuador. (lunaf3@hotmail.com; renataleon@yahoo.com; vrafael@puce.edu.ec; diego cesp@hotmail.com)

\begin{abstract}
Four new species of the Drosophila onychophora species group (Diptera, Drosophilidae) in the Parque Arqueológico Rumipamba, Pichincha, Ecuador. The species of the subgenera Phloridosa and Drosophila (in this last one, regarding the Drosophila species groups: D. flavopilosa, D. onychophora and D. bromeliae) are species of Drosophila that developed only in flowers. The group D. onychophora was proposed to bring together exclusively antophilic species. The former group is composed by 18 species, and is associated to flowers of Asteraceae. Captures were made in the Parque Arqueológico Rumipamba, Pichincha, Ecuador, during February, May, July, September and December of 2009. The species were collected directly from flowers with an insect aspirator. A total of 427 individuals from Drosophila were captured. We identified 11 species, of which six are new species of the $D$. onychophora species group and one is a new record for Ecuador. In this paper we describe four of the six new species.
\end{abstract}

KEYWORDS. Antophilic, Asteraceae, monophagous, neotropical, new record.

RESUMEN. Las especies de los subgéneros Phloridosa y Drosophila (este último en relación con los grupos de especies: D. flavopilosa, D. onychophora y D. bromeliae) son especies de Drosophila que se desarrollan únicamente en flores. El grupo D. onychophora fue propuesto para agrupar a especies exclusivamente antófilas. El grupo D. onychophora está integrado por 18 especies, que se encuentran relacionadas con las flores de Asteraceae. En el Parque Arqueológico Rumipamba, provincia de Pichincha, Ecuador, se realizaron capturas de Drosophila, en los meses de febrero, mayo, julio, septiembre y diciembre del 2009. Las especies fueron recolectadas directamente en las flores con un aspirador entomológico. En total se capturaron 427 individuos de Drosophila y se identificaron 11 especies, de las cuales seis son nuevas especies del grupo D. onychophora y una es un nuevo registro para el Ecuador. En este trabajo se describen cuatro de las seis especies nuevas.

PALABRAS-CLAVE. Antófila, Asteraceae, monófaga, neotropical, nuevo registro.

Los insectos desempeñan en los bosques funciones muy diversas e importantes tales como fitófagos, descomponedores, polinizadores, depredadores, parásitos, o vectores de organismos patógenos (DAJOZ, 1999). Muchas especies de insectos holometábolos utilizan sustratos efímeros (hongos, flores, frutos, etc.) para su desarrollo y alimentación en los periodos de larva y de adulto. Entre las drosófilas que se alimentan de flores existen varias especies que están asociadas con una sola planta hospedera (monófagas). Otras especies se alimentan de dos especies de plantas e incluso existen algunas especies que se alimentan y reproducen en varias flores diferentes (polífagas) (PIPKIN et al., 1966; dos Santos \& Vilela, 2005). En la región Neotropical, el género Drosophila está altamente diversificado ( $\mathrm{VAL}$ et al., 1981). Un factor puede ser que, en estas regiones el periodo de floración es largo y además existe una amplia diversidad de flora (PIPKIN et al., 1966).

STURTEVANT (1921) fue el primero en reportar que Drosophila (Drosophila) florae (grupo bromeliae) y Drosophila (Phloridosa) lutzii, se encontraban en flores del género Datura (Solanaceae) y algunas especies de Cucumis (Cucurbitaceae) (BRNCIC, 1983). BRnCIC (1966) también observó una asociación entre los miembros del grupo D. flavopilosa con diferentes especies de Cestrum (Solanaceae). Así mismo, Hunter (1979) encontró que varias especies antofílicas estaban relacionadas con las flores de la familia Asteraceae, posteriormente estas especies fueron reunidas en el grupo $D$. onychophora por VILELA \& BäChli (1990). Por otro lado las especies del grupo $D$. bromeliae son más generalistas y han sido reportadas en flores de Convolvulaceae, Pontederiaceae, Aristolochiaceae y Malvaceae (SILVA \& MARTINS, 2004). Las especies del subgénero Phloridosa y de los grupos de especie Drosophila flavopilosa, Drosophila onychophora y Drosophila bromeliae del subgénero Drosophila, son las únicas del género que se desarrollan exclusivamente en flores (VELA \& RAFAEL, 2004).

Las características de las especies del grupo $D$. onychophora son: oviscapto de las hembras con muchos dientes pequeños y los huevos no poseen filamentos, lo que sugiere una relación con las especies del subgénero Phloridosa, aunque probablemente su evolución haya sido independiente (VILEla \& Bächli, 1990; Hunter, 1992). El grupo $D$. onychophora agrupa a 18 especies: D. acuminanus Hunter, 1988; D. arane Hunter, 1992; D. arboloco Hunter, 1979; D. bifurcada Hunter, 1992; D. bomarea Hunter, 1979; D. carablanca Hunter, 1979; D. chisaca Hunter, 1988; D. choachi Hunter, 1992; D. colmenares Hunter, 1988; D. desbaratabaile Hunter, 1979; D. franii Hunter, 1979; D. freilejoni Hunter, 1979; D. hyalipennis Duda, 1927; D. margarita Hunter, 1979; D. onychophora Duda, 1927; D. pulverea Duda, 1927 (Vilela \& Bächli, 1990; Hunter, 1992) y D. yurag Figuero \& Rafael, 2011, D. yuragshina Figuero \& Rafael, 2011 (Figuero \& RAFAel, 2011). 
En el presente estudio se describen cuatro nuevas especies del grupo $D$. onychophora capturadas con aspirador entomológico de flores compuestas en el Parque Arqueológico Rumipamba en Quito, Ecuador. Estos datos corroboran la asociación de este grupo de Drosophila a plantas de Asteraceae y revela nuevos datos sobre la diversidad del género en el país.

\section{MATERIALES Y MÉTODOS}

Las capturas de las especies del género Drosophila se llevaron a cabo en el Parque Arqueológico Rumipamba, provincia de Pichincha, Distrito Metropolitano de Quito, Ecuador. La altitud del parque va desde los $2.885 \mathrm{~m}$ a los $2.930 \mathrm{~m}$

En el parque se establecieron cuatro transectos de $50 \mathrm{~m}$ de longitud cada uno. El primer transecto se encuentra en la quebrada Rumipamba, $2.903 \mathrm{~m}$ de altitud, $00^{\circ} 10^{\prime} 52.5^{\prime \prime} \mathrm{S}, \quad 78^{\circ} 30^{\prime} 00.1^{\prime \prime} \mathrm{W}$. El segundo transecto está ubicado en la entrada al "colunco", 2.924 $\mathrm{m}, 00^{\circ} 10^{\prime} 43.4^{\prime \prime} \mathrm{S}, 78^{\circ} 30^{\prime} 08.1^{\prime \prime} \mathrm{W}$. El tercer transecto se encuentra detrás de la casa hacienda, $2.921 \mathrm{~m}$, $00^{\circ} 10^{\prime} 49.9^{\prime \prime} \mathrm{S}, \quad 78^{\circ} 30^{\prime} 04.8^{\prime \prime} \mathrm{W}$. El cuarto transecto está localizado delante de la casa hacienda, $2.914 \mathrm{~m}$, $00^{\circ} 10^{\prime} 49.0^{\prime \prime} \mathrm{S}, 78^{\circ} 30^{\prime} 04.8^{\prime \prime} \mathrm{W}$.

Se realizaron muestreos en los meses de febrero, mayo, julio, septiembre y diciembre del 2009. Las drosófilas fueron recolectadas directamente de las flores con un aspirador entomológico. En los dos primeros transectos, la recolección se realizó en todas las flores presentes, mientras que en el tercer transecto, se muestreo únicamente en flores de Pappobolus imbaburensis (Hieron.) Panero y en el cuarto transecto, sólo en flores de Verbesina sodiroi Hieron., ambas de la familia Asteraceae.

La identificación de las especies se hizo mediante el análisis de la morfología externa y de la genitalia. Se utilizó BÄCHLI et al. (2004) como referencia para nombrar las estructuras en las descripciones de las especies, así como para los índices alares.

Los holotipos y paratipos se montaron en seco pegados en triángulos de cartulina y la genitalia se colocó en un microtubo con glicerol. Todos los individuos están depositados en el Museo de Zoología, sección Invertebrados de la Pontificia Universidad Católica del Ecuador, Quito (QCAZ-I).

\section{RESULTADOS}

En total se capturaron 427 individuos de Drosophila y se identificaron 11 especies y dos morfotipos de hembras. Seis de ellas corresponden a especies nuevas del grupo $D$. onychophora. También se capturó a D. arane en flores de Baccharis sp. ("chilca"); esta especie miembro del grupo $D$. onychophora es un nuevo registro para el Ecuador. Además se identificaron a D. ogradi Vela \& Rafael, 2004 (grupo D. morelia); D. ecuatoriana Vela \& Rafael, 2004 (grupo D. guarani) y D. ninarumi Vela \& Rafael, 2005 (grupo D. tripunctata).

\section{Drosophila verbesinae sp. nov.}

(Figs 1-7)

Material tipo. Holotipo $\widehat{\partial}$ (disectado, montado en seco, genitalia en microtubo), Ecuador, Pichincha, Quito (Parque Arqueológico Rumipamba, 00¹0'49.9”S, 78³0’04.8”'W, 2.914 m), II.2009, R. León col., R. León det. (QCAZI 2476); alotipo 9 (disectado, montado en seco, genitalia en microtubo), los mismos datos del holotipo (QCAZI 2487); paratipos: 10 $\hat{0}, 9$ ( 9 (disectados, montados en seco, genitalia en microtubo), los mismos datos del holotipo (QCAZI 2477-2486, 2488-2496)

Diagnosis. Cuerpo marrón oscuro. Tórax con pelos amarillos. Cerdas escutelares anteriores divergentes. Abdomen con línea media dorsal a partir del tercer tergito. La hembra es igual pero más oscura y sin línea media dorsal en el abdomen. Cerci libres, cabeza del edeago en forma de $\mathrm{C}$ y con el extremo distal quitinizado en forma de pico. Oviscapto con 100 a 140 dientes.

Descripción del macho, holotipo y paratipos (individuos muertos secos). Morfología externa. Cuerpo oscuro brillante polinoso. Longitud del cuerpo del holotipo incluido alas: $3,00 \mathrm{~mm}$. Longitud del cuerpo de los paratipos incluido alas: 2,50 - 3,10 $\mathrm{mm}$.

Cabeza. Color marrón oscura. El primero y segundo segmento de las antenas marrón, arista plumosa marrón amarillenta, presenta 3 ramas dorsales y 1 ventral más la terminal bifurcada. Placa orbital negra, con la cerda orbital media muy cercana al borde exterior de la placa orbital y más cerca a la cerda orbital anterior. Triángulo ocelar marrón, ocelos amarillos. Triángulo frontal y frontal vitta de color negro. Carina marrón oscura, no muy prominente, no surcada, gena marrón oscuro. Palpos con 2 cerdas de color marrón claro. Ojos marrón oscuro.

Tórax. Color marrón oscuro, polinoso (algunos individuos presentan el tórax más claro), con pelos y cerdas amarillentas, 6 hileras de pelos acrosticales entre las cerdas dorsocentrales anteriores, escutelo polinoso, cerdas escutelares anteriores divergentes. Cerda esternopleural media $1 / 3$ de la anterior. Patas amarillas, fémur ligeramente más oscuro, el quinto segmento tarsal marrón amarillento. Alas amarillentas, longitud de las alas $2,10 \mathrm{~mm}$, ancho $1,00 \mathrm{~mm}$. Índices alares: $C=4,10 ; a c=1,66 ; \mathrm{hb}=0,50 ; 4 \mathrm{C}=0,71 ; 4 \mathrm{v}=2,07$; $5 \mathrm{x}=1,60 ; \mathrm{M}=0,57 ;$ Prox $\mathrm{x}=0,57$.

Abdomen. Color marrón, la parte inferior de cada tergito más oscura, con línea media dorsal, la misma que aparece a partir del tercer tergito.

Genitalia externa. Cerci libres, micropubescentes y con cerdas; epandrio con finos pelos, lóbulo ventral con 10 cerdas largas en el lado derecho y 9 en el izquierdo (en otros individuos de 8 a 12) y 7 cerdas más cortas en la prolongación de la parte baja anterior que es larga y curva (en otros individuos de 6 a 8 cerdas cortas). 
Surestilo de forma rectangular, micropubescente hacia el lado interno y no micropubescente hacia el lado externo, presenta 8 dientes primarios y 4 cerdas marginales (en otros individuos de 7 a 9 dientes primarios y de 4 a 5 cerdas marginales). Decasternum en forma de arco (Fig. 1).

Genitalia interna. Hipandrio más corto que el epandrio, en forma de $\mathrm{U}$, con el borde externo más quitinizado. Parte anterior del hipandrio triangular. Gonopodios membranosos y cóncavos, en la parte inferior presentan una cerda larga (Fig. 2). Edeago curvo y doblado hacia la parte ventral (en forma de C), el extremo distal es más quitinizado y termina en punta (en forma de pico), la punta dorsal del edeago semeja un moñón, con ligeras ondulaciones; el edeago es abierto a lo largo de la parte ventral y en la parte dorsal presenta una doble línea sin ser abertura. El apodema del edeago es recto y menos quitinizado, la parte más baja es membranosa y presenta estrías. Paráfisis alargada y triangular, en la parte apical presenta 2 cerdas. La rama ventral es alargada. Índice del edeago 1,70 (Figs 3-5).

Descripción de la hembra, alotipo y paratipos (individuos muertos en alcohol). Morfología externa. Cuerpo brillante polinoso. Longitud del cuerpo del alotipo incluido alas: 2,90 mm. Longitud del cuerpo de los paratipos incluido alas: 2,90 - 3,10 $\mathrm{mm}$.

La hembra presenta las mismas características que el macho, excepto las patas que son más oscuras, y el abdomen es más oscuro y no presenta línea media dorsal. Longitud de las alas de un paratipo + (QCAZ 2494) $2,36 \mathrm{~mm}$, ancho $1,05 \mathrm{~mm}$. Índices alares tomados del mismo paratipo ?: $\mathrm{C}=3,66 ; \mathrm{ac}=2,00 ; \mathrm{hb}=0,33$; $4 \mathrm{C}=0,66 ; 4 \mathrm{v}=1,61 ; 5 \mathrm{x}=1,16 ; \mathrm{M}=0,38 ;$ Prox $\mathrm{x}=0,50$.

Genitalia. Oviscapto muy quitinizado, de color marrón; posee 137 dientes, pequeños y gruesos (en otros individuos de 100 a 140 dientes), sin dientes en la parte anterior; presenta 4 cerdas en la parte posterior, 3 en la parte ventral y una cerda más larga en la parte dorsal (Fig. 6). Espermatecas pequeñas, muy quitinizadas, ovaladas y porosas. Índice de la espermateca 0,90 (Fig. 7).

Semejanzas morfológicas con otras especies. Drosophila verbesinae sp. nov. se asemeja mucho en la genitalia a $D$. freilejoni, del grupo $D$. onychophora, $\sin$ embargo también presentan algunas diferencias y son especies asociadas a diferentes plantas hospederas.

Planta hospedera. Todos los individuos fueron capturados en flores de Verbesina sodiroi (Asteraceae).

Etimología. El nombre Drosophila verbesinae sp. nov. hace referencia al género de la planta hospedera, Verbesina sodiroi.

\section{Drosophila pappobolusae sp. nov.}

(Figs 8-14)

Material tipo. Holotipo $\hat{\delta}$ (disectado, montado en seco, genitalia en microtubo), Ecuador, Pichincha, Quito (Parque Arqueológico Rumipamba, 00¹0'52.5”S, 78³0’00.1”W, 2.903 m), II.2009, R. León col., R. León det. (QCAZI 2497); alotipo (disectado, montado en seco, genitalia en microtubo), los mismos datos del holotipo (QCAZI 2508); paratipos: 10仓̂, $10 q$ (disectados, montados en seco, genitalia en microtubo), los mismos datos del holotipo (QCAZI 2498-2507, 2509-2518).

Diagnosis. Cuerpo marrón oscuro. Cerdas escutelares anteriores divergentes. Abdomen sin línea media dorsal. Cerci libres. Edeago en forma de C, distalmente muy quitinizado semejante a un martillo. Oviscapto quitinizado, con 80 a 110 dientes.

Descripción del macho, holotipo (individuo vivo) y paratipos (individuos muertos en alcohol). Morfología externa. Cuerpo oscuro brillante polinoso. Longitud del cuerpo del holotipo incluido alas: 3,00 $\mathrm{mm}$. Longitud del cuerpo de los paratipos incluido alas: $3,00-3,20 \mathrm{~mm}$.

Cabeza. Color marrón. El primer y segundo segmentos de las antenas marrón oscuros, arista plumosa marrón amarillenta, presenta 3 ramas dorsales y 1 ventral más la terminal bifurcada. Placa orbital negra, cerda orbital media muy cercana al borde exterior de la placa orbital y más cerca a la cerda orbital anterior. Triángulo ocelar polinoso, ocelos color marrón claro. Triángulo frontal y frontal vitta de color negro. Carina marrón claro, prominente no surcada, gena marrón claro. Palpos amarillentos. Ojos color marrón.

Tórax. Color marrón oscuro; 6 hileras de pelos acrosticales entre las cerdas dorsocentrales anteriores, escutelo del mismo color, cerdas escutelares anteriores divergentes. Cerda esternopleural media $1 / 3$ de la anterior. Patas amarillas, excepto la coxa y el último segmento tarsal que son marrón oscuro. Alas transparentes, longitud de las alas $2,25 \mathrm{~mm}$, ancho $1,00 \mathrm{~mm}$. Índices alares: $\mathrm{C}=3,00 ; \mathrm{ac}=2,50 ; \mathrm{hb}=0,40 ; 4 \mathrm{C}=0,77 ; 4 \mathrm{v}=1,70$; $5 \mathrm{x}=1,31 ; \mathrm{M}=0,44$; Prox $\mathrm{x}=0,36$.

Abdomen. Color amarillo, sin línea media dorsal, los dos primeros tergitos marrones amarillentos, el tercer tergito marrón amarillento en la parte media y luego se adelgaza hacia los lados, la parte baja de los demás tergitos con pigmentación marrón oscura.

Genitalia externa. Cerci libres, micropubescentes y con cerdas, epandrio con finos pelos, en el lóbulo ventral presenta 14 cerdas largas y cortas, las cortas en la parte más baja (en otros individuos de 10 a 16 cerdas). Surestilo ovalado, desnudo, estriado, con 4 dientes primarios a cada lado (en otros individuos de 4 a 6 dientes) y 4 cerdas marginales hacia la cara interna de los dientes primarios, no distribuidas uniformemente (en otros individuos de 3 a 4 cerdas). Decasternum en forma de $\operatorname{arco}$ (Fig. 8).

Genitalia interna. Hipandrio más corto que el epandrio, en forma de $\mathrm{U}$, el borde externo más quitinizado. Parte anterior del hipandrio pequeña, gonopodios delgados y poco quitinizados (Fig. 9). Edeago quitinizado, en forma de $\mathrm{C}$, la parte apical 


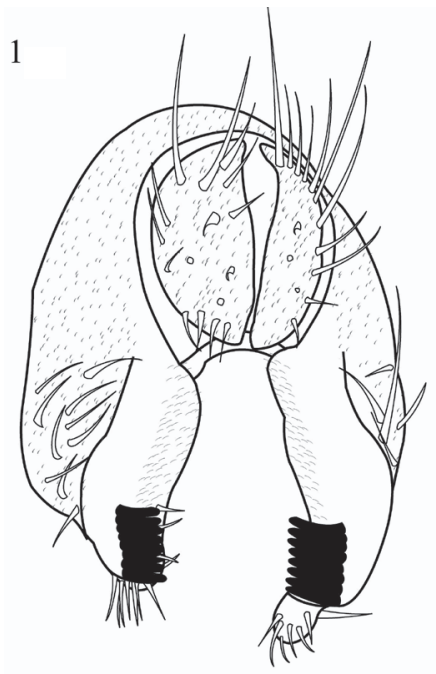

100um
2

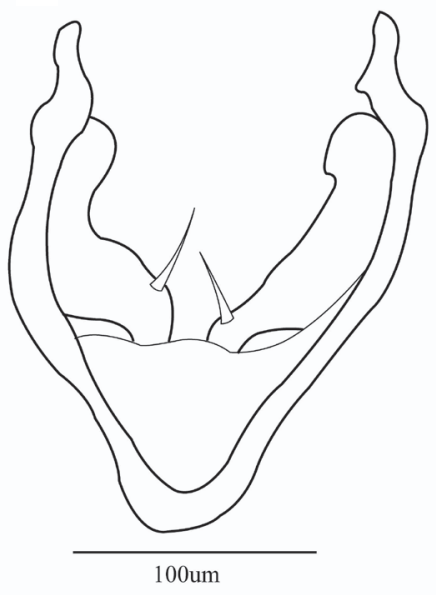

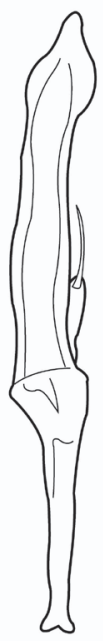

4

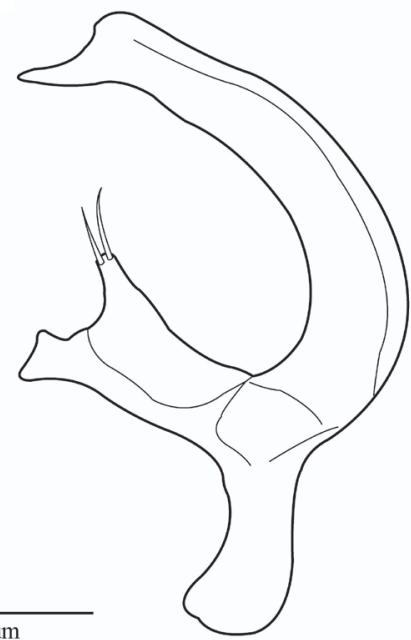

5

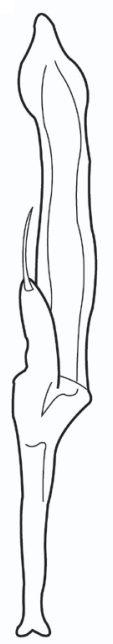

6

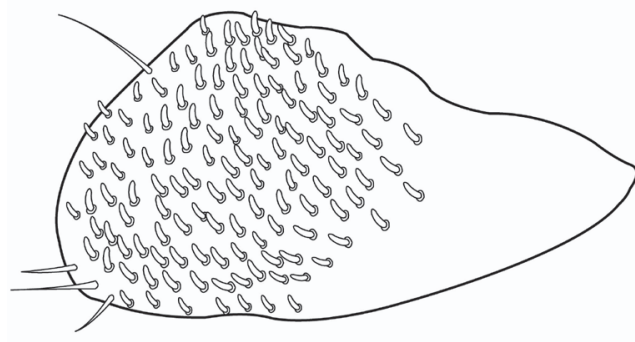

7

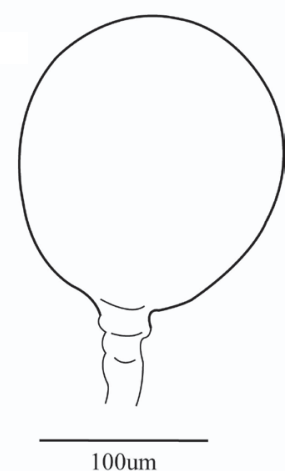

Figs 1-7. Drosophila verbesinae sp. nov., holotipo ô: 1, cerci; 2, hipandrio; edeago: 3, vista dorsal; 4, vista lateral; 5, vista ventral. Alotipo O $_{\text {: }} 6$, oviscapto; 7, espermateca.

sumamente quitinizada y semeja a un martillo o a una T, el extremo distal más largo, delgado, quitinizado y termina en punta, mientras que la parte baja (el otro lado de la T) es más corta, menos quitinizada y roma, el ápice del edeago es abierto ventralmente hasta muy cerca del extremo apical, en el lado dorsal sin ninguna hendidura, el apodema del edeago recto y quitinizado excepto la parte más baja que es membranosa y estriada. Paráfisis distal de forma triangular y en el extremo distal con 2 cerdas. Índice del edeago 2,00 (Figs 10-12). Testículos amarillos, dos vueltas externas y una vuelta interna.

Descripción de la hembra, alotipo (individuo vivo) y paratipos (individuos muertos en alcohol). Morfología 
externa. Cuerpo oscuro brillante polinoso. Longitud del cuerpo del alotipo incluido alas: 3,20 mm. Longitud del cuerpo de los paratipos incluido alas: 3,00 - 3,20 mm.

La hembra con las mismas características del macho pero más oscura. Longitud de las alas de un paratipo + (QCAZ 2510) 2,03 $\mathrm{mm}$, ancho 0,94 mm. Índices alares tomados del mismo paratipo $\circ$ : $\mathrm{C}=3,63$; $\mathrm{ac}=2,20 ; \mathrm{hb}=0,27 ; 4 \mathrm{C}=0,78 ; 4 \mathrm{v}=2,07 ; 5 \mathrm{x}=1,60 ; \mathrm{M}=0,57$; Prox $\mathrm{x}=0,71$. Genitalia. Oviscapto quitinizado, con 99 dientes pequeños y gruesos, sin dientes en la parte

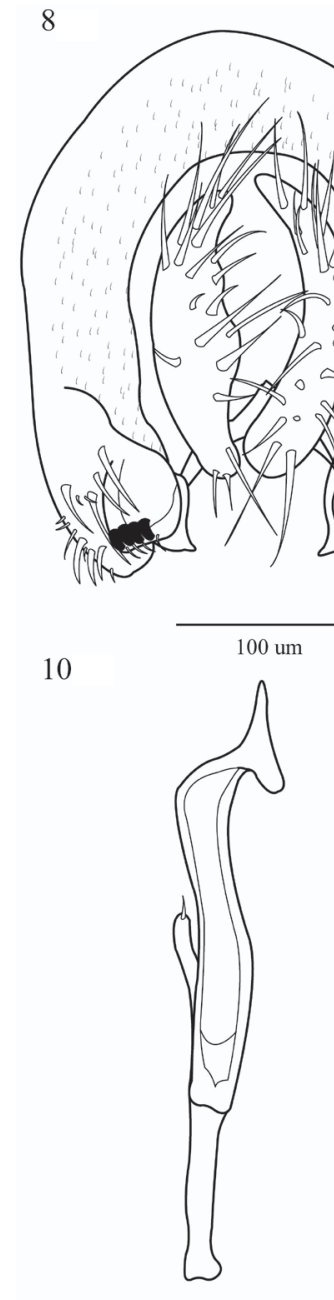

13

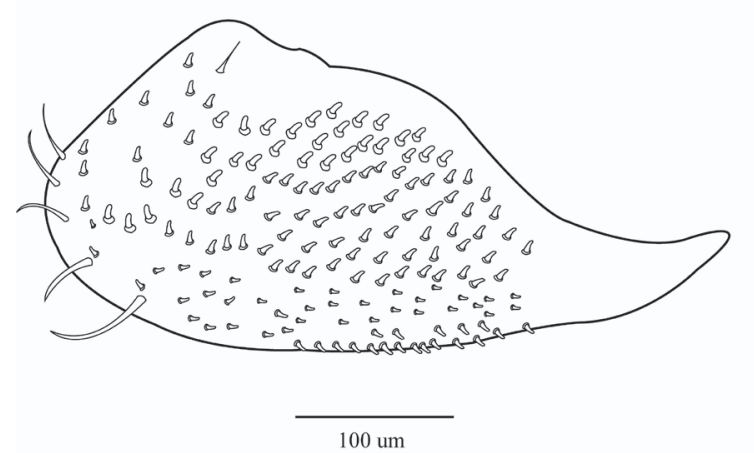

anterior (en otros individuos de 80 a 110 dientes), posee 5 cerdas en la parte posterior (Fig. 13). Espermatecas muy quitinizadas, ovaladas, pequeñas y porosas. Índice de la espermateca 0,80 (Fig. 14).

Planta hospedera. Todos los individuos fueron capturados en flores de Pappobolus imbaburensis (Asteraceae).

Etimología. El nombre Drosophila pappobolusae sp. nov. hace referencia al género de la planta hospedera, Pappobolus imbaburensis.

9

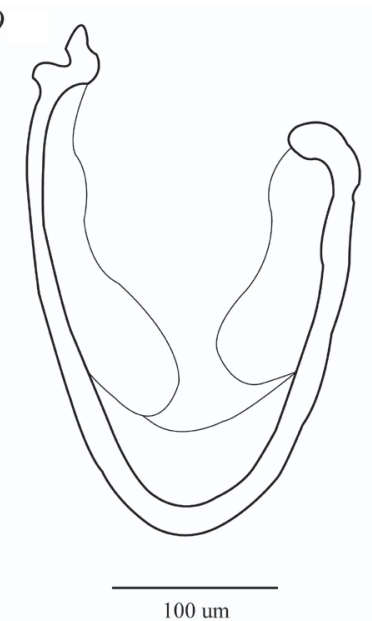

12

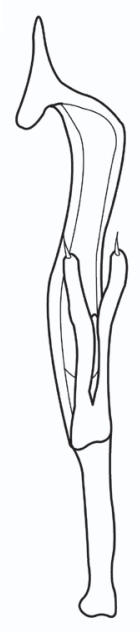

14

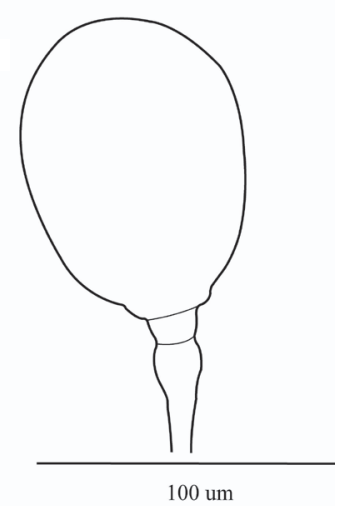

Figs 8-14. Drosophila pappobolusae sp. nov., holotipo ồ : 8, cerci; 9, hipandrio; edeago: 10, vista dorsal; 11, vista lateral; 12, vista ventral. Alotipo +: 13 , oviscapto; 14 , espermateca. 


\section{Drosophila sisapamba sp. nov.}

(Figs 15-19)

Material tipo. Holotipo $\widehat{\partial}$ (disectado, montado en seco, genitalia en microtubo), Ecuador, Pichincha, Quito (Parque Arqueológico Rumipamba, $00^{\circ} 10^{\prime} 52.5^{\prime}$ 'S, 78³0’00.1”W, $2.903 \mathrm{~m}$ ), V.2009, R. León col., R. León det. (QCAZI 2543); paratipos: 23 (disectados, montados en seco, genitalias en microtubo), los mismos datos del holotipo (QCAZI 2544, 2545).

Diagnosis. Cuerpo marrón oscuro. Cerdas escutelares anteriores paralelas. Abdomen con línea media dorsal. Primer y segundo tergito oscuros. Cerci libres. Edeago quitinizado en forma de C. El extremo distal-ventral de la cabeza del edeago con una pequeña proyección a manera de gancho y en la parte mediaventral otra proyección como gancho más grande.

Descripción del macho, holotipo y paratipos (individuos muertos en seco). Morfología externa. Cuerpo marrón oscuro. Longitud del cuerpo del holotipo incluido alas: 3,00 mm. Longitud del cuerpo de los paratipos incluido alas: $2,80-3,00 \mathrm{~mm}$.

Cabeza. Color marrón oscuro. Primer y segundo segmento de las antenas marrón claro, arista plumosa con 2 ramas dorsales y 1 rama ventral, más la rama terminal bifurcada. Placa orbital marrón oscura, la cerda orbital media más cerca a la orbital anterior y cercana al borde ocular. Triángulo ocelar marrón oscuro, ocelos de color naranja. Frontal vitta marrón oscura. Carina marrón clara, no surcada, gena marrón. Proboscis amarillenta, palpos con 2 cerdas grandes. Ojos marrón oscuros.

Tórax. Color marrón oscuro, con 6 hileras de pelos acrosticales entre las cerdas dorsocentrales anteriores, escutelo marrón oscuro, cerdas escutelares anteriores paralelas. Cerdas esternopleurales rotas. Patas amarillas, último segmento tarsal ligeramente más oscuro. Alas amarillentas claras, longitud de las alas $3,00 \mathrm{~mm}$. Índices alares: $\mathrm{C}=4,00 ; \mathrm{ac}=1,70 ; \mathrm{hb}=0,36 ; 4 \mathrm{C}=1,10 ; 4 \mathrm{v}=5,00$; $5 \mathrm{x}=1,40 ; \mathrm{M}=0,70 ;$ Prox $\mathrm{x}=1,00$.

Abdomen. Primero y segundo segmentos abdominales oscuros, el tercer, cuarto y quinto tergitos exhiben una banda oscura a lo largo del borde inferior. Presenta línea media dorsal.

Genitalia externa. Cerci libres, con cerdas y pelos, epandrio con pelos, en la parte inferior con cerdas, lóbulo ventral delgado con 11 cerdas a cada lado (en otros individuos de 9 a 11 cerdas). Surestilo alargado, verrugoso con 8 dientes primarios (en otros individuos de 7 a 8 dientes). Presenta 8 cerdas marginales en el lado derecho y 10 cerdas marginales en el izquierdo. Decasternum quitinizado, en forma de $\operatorname{arco}$ (Fig. 15).

Genitalia interna. Hipandrio más largo que el epandrio, en forma de U, ligeramente quitinizado, parte anterior del hipandrio pequeña, gonopodios alargados, ligeramente quitinizados con los bordes membranosos (Fig. 16). Edeago quitinizado en forma de $\mathrm{C}$, distalmente curvado y doblado hacia la parte ventral con una pequeña proyección quitinizada a manera de gancho, dirigida hacia la parte posterior. La parte distal del edeago termina en punta con pequeñas ondulaciones ( 2 a 3 ) en la parte anterior. En la parte media-ventral presenta otra estructura quitinosa a manera de gancho, la misma que está unida a la cabeza del edeago a través de una membrana. Apodema curvo, poco quitinizado con estrías en la parte terminal. Paráfisis larga en forma de dedo, con 4 cerdas en la punta (muy juntas en pares). Rama ventral laminar, larga y cóncava. Índice del edeago 3,00 (Figs 17-19).

Etimología. El nombre Drosophila sisapamba sp. nov. hace referencia a las palabras quichuas sisa, flor y pamba, prado o llanura. Esta especie fue capturada en el Parque Arqueológico Rumipamba.

\section{Drosophila wayta sp. nov.}

(Figs 20-24)

Material tipo. Holotipo o (disectado, montado en seco, genitalia en microtubo), Ecuador, Pichincha, Quito (Parque Arqueológico Rumipamba, 00¹0'52.5”S, 78³0'00.1”W, $2.903 \mathrm{~m}$ ), II.2009, R. León col., R. León det. (QCAZI 2546); paratipos: 43 (disectados, montados en seco, genitalias en microtubo), los mismos datos del holotipo (QCAZI 2547 - 2550).

Diagnosis. Cuerpo marrón oscuro. Tórax polinoso, cerdas escutelares anteriores divergentes. Abdomen amarillo, primero y segundo tergitos oscuros. Cerci libres. Parte distal del edeago crestada (clavel) y bífida.

Descripción del macho, holotipo y paratipos (individuos muertos en alcohol). Morfología externa. Cuerpo marrón oscuro. Longitud del cuerpo del holotipo incluido alas: 3,00 $\mathrm{mm}$. Longitud del cuerpo de los paratipos incluido alas: $2,80-3,10 \mathrm{~mm}$.

Cabeza. Los dos primeros segmentos de las antenas oscuros, arista plumosa con tres ramas dorsales y dos ventrales, más la terminal bifurcada. Cabeza marrón oscura. Placa orbital marrón oscura, la cerda orbital media más cerca a la orbital anterior. Triángulo ocelar oscuro, ocelos amarillos. Frontal vitta marrón clara. Carina amarillenta, no prominente, no surcada, gena marrón clara, cara amarilla. Proboscis amarillo. Ojos rojo vinoso.

Tórax. Polinoso, 6 hileras de pelos acrosticales entre las cerdas dorsocentrales anteriores, tórax y escutelo marrón oscuros, cerdas escutelares anteriores largas y divergentes. Cerda esternopleural media $1 / 2$ de la anterior. Patas amarillas, quinto segmento tarsal marrón oscuro. Alas amarillentas transparentes, longitud de las 


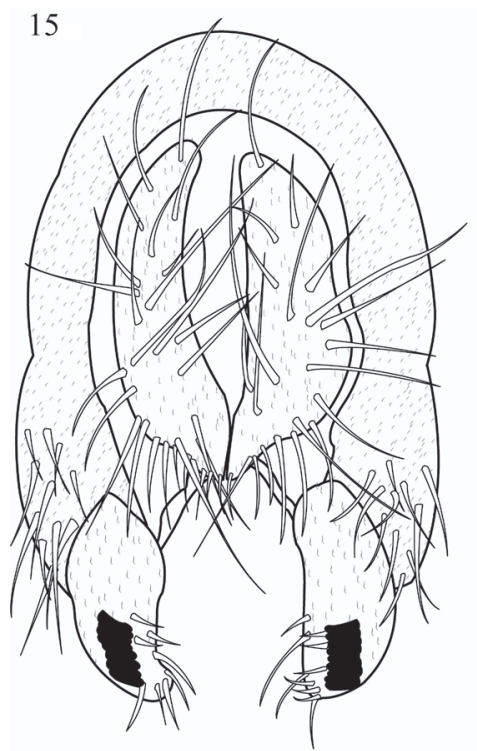

$100 \mathrm{um}$
16

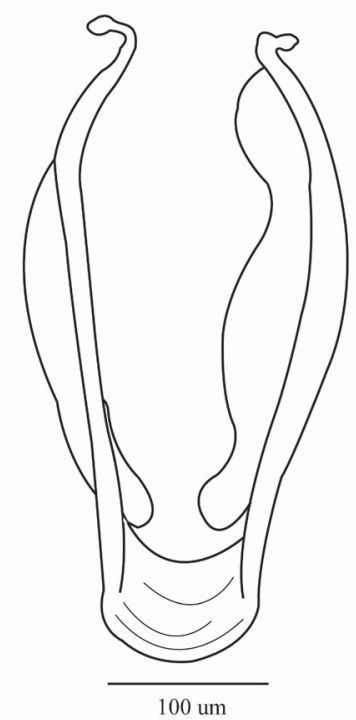

17

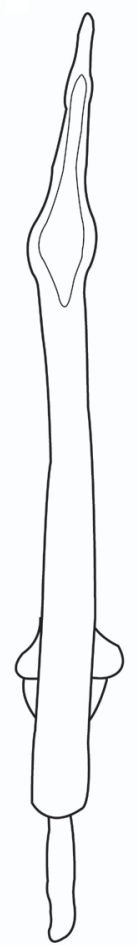

18

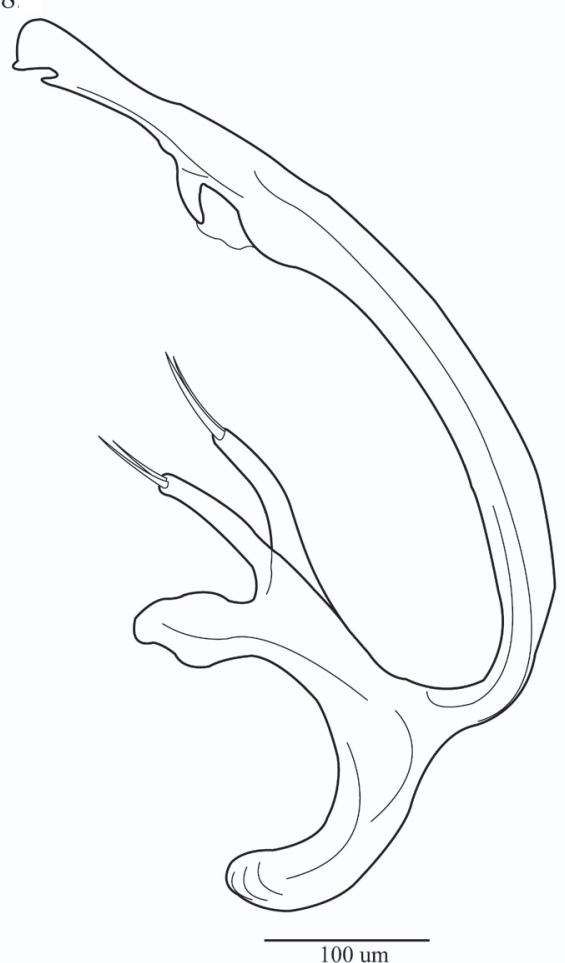

19

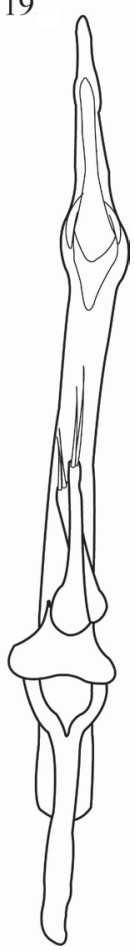

Figs 15-19. Drosophila sisapamba sp. nov., holotipo ${ }^{\Uparrow}: 15$, cerci; 16, hipandrio; edeago: 17, vista dorsal; 18, vista lateral; 19 , vista ventral.

alas $2,50 \mathrm{~mm}$, ancho $1,00 \mathrm{~mm}$. Índices alares: $\mathrm{C}=4,20$; $\mathrm{ac}=1,66 ; \mathrm{hb}=0,40 ; 4 \mathrm{C}=0,66 ; 4 \mathrm{v}=1,93 ; 5 \mathrm{x}=1,80 ; \mathrm{M}=0,60$; Prox $\mathrm{x}=0,53$.

Abdomen. Color amarillento, excepto el primero y segundo segmentos que son más oscuros.

Genitalia externa. Cerci libres, con cerdas y pelos, termina en punta. Epandrio sumamente peludo. Lóbulo ventral derecho con 9 cerdas en el lado derecho y 8 en lado izquierdo (en otros individuos de 8 a 10 cerdas). Surestilo ovalado, con 11 dientes primarios en el lado derecho y 12 en el izquierdo (en otros individuos de 8 a 12 dientes), 13 cerdas marginales en el lado derecho y 13 en el izquierdo (en otros individuos de 7 a 12 cerdas). Decasternum altamente quitinizado, en forma de arco (Fig. 20).

Genitalia interna. Hipandrio más largo que el epandrio, prolongado y delgado, en forma de $\mathrm{U}$, los 
márgenes laterales quitinizados, parte anterior del hipandrio membranosa, gonopodios membranosos, con una cerda larga en la parte interna (Fig. 21). Edeago quitinizado recto con la punta crestada (clavel) y bífida. Presenta dos ramas en forma de pirámide que alcanzan $1 / 2$ del ápice del edeago. Entre las ramas y el ápice del edeago hay una estructura membranosa. Paráfisis rectangulares, alcanzan $3 / 4$ de las ramas. Apodema del edeago largo, la parte distal es membranosa. Índice del edeago 1,25 (Figs 22-24).

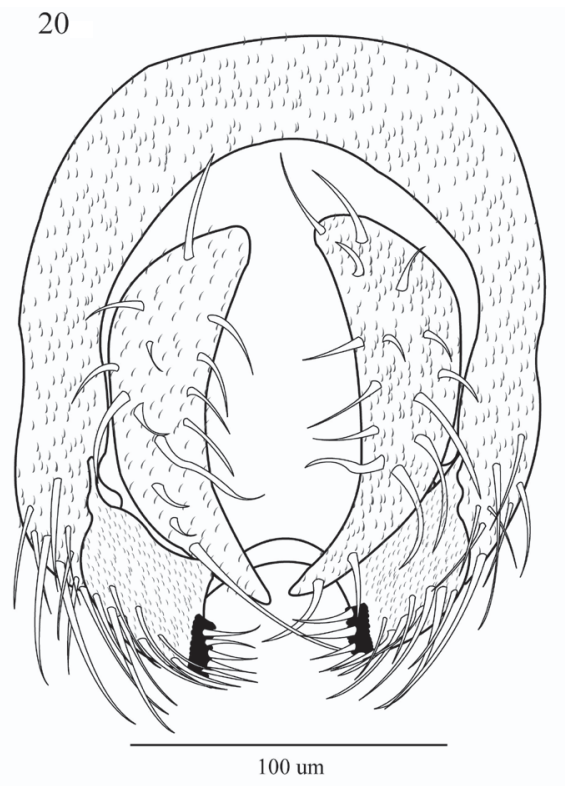

22

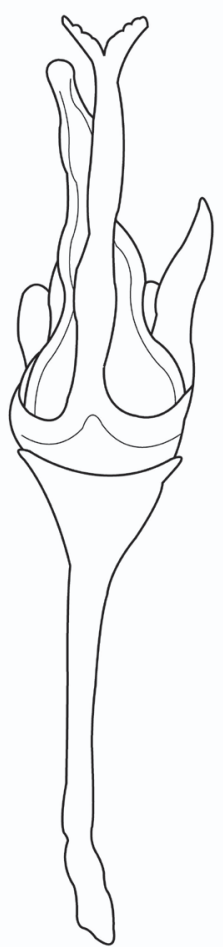

Etimología. El nombre Drosophila wayta sp. nov. hace referencia a la palabra quichua wayta que significa clavel, ya que la cabeza del edeago del macho de esta especie es aserrada y se asemeja a un clavel.

\section{DISCUSIÓN}

Las especies de Drosophila asociadas a las flores han sido poco estudiadas ya que raramente son capturadas con red o con trampas de fruta fermentada (PIPKIN et al.,

21

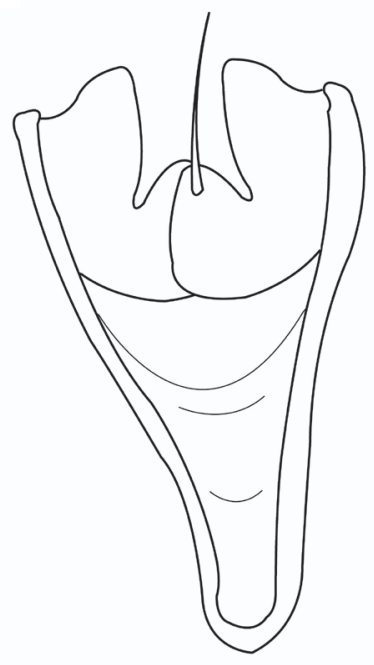

100 um

24
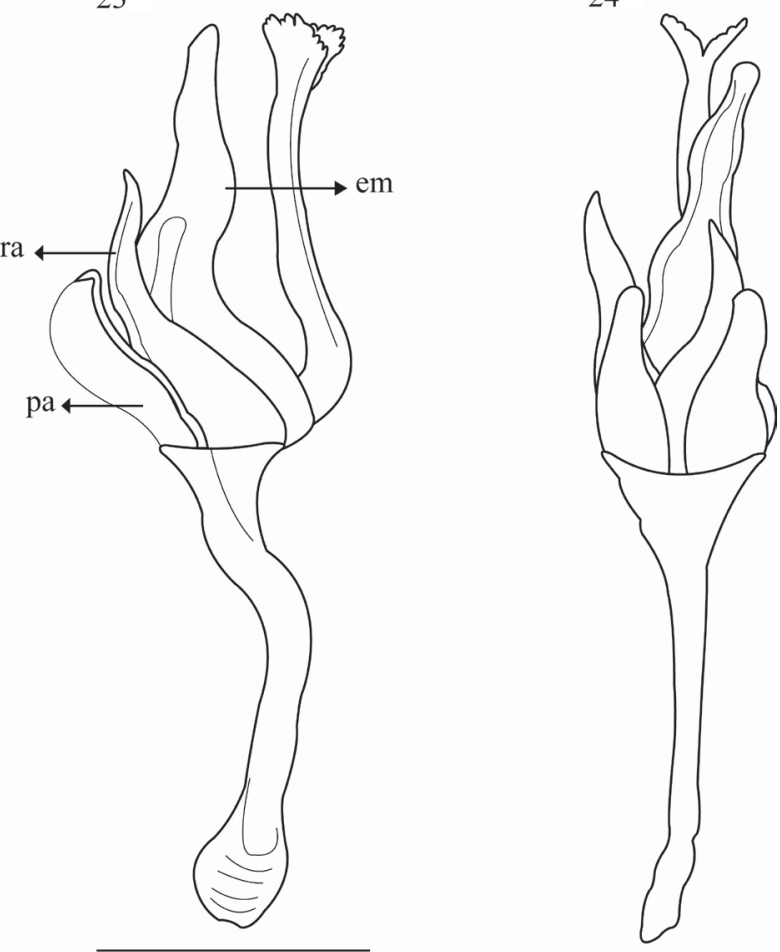

Figs 20-24. Drosophila wayta sp. nov., holotipo ô: 20, placa anal; 21, hipandrio; edeago: 22, vista dorsal; 23, vista lateral; 24, vista ventral (em, estructura membranosa; pa, paráfisis; ra, ramas). 
1966); la única manera de capturarlas es directamente de las flores. Además estas especies asociadas a flores son muy difíciles de reproducir en laboratorio debido a sus exigencias alimenticias específicas.

El grupo D. onychophora presenta características morfológicas únicas y determinantes; una de ellas es la morfología del oviscapto (VILELA \& Bächli, 1990). El análisis de esta estructura es indispensable para incorporar a las especies dentro del grupo $D$. onychophora. Aunque la forma y el número de dientes del ovipositor son determinantes, la forma del edeago también es muy importante. Las moscas de flores tienen características relacionadas a su hábitat particular y la adaptación de estas características es más evidente en especies que muestran una extrema especificidad con el hospedero (BRNCIC, 1983). En este trabajo se identificaron a dos hembras de las cuatro especies descritas; sin embargo las otras dos especies presentan edeagos alargados propios del grupo y de las especies del subgénero Drosophila asociadas a flores (VILELA \& BäCHLI, 1990 y HunTER, 1992).

Drosophila verbesinae sp. nov. y D. pappobolusae sp. nov. son consideradas del grupo D. onychophora ya que las hembras de estas dos especies presentan ovipositores propios del grupo. Además varios estudios han demostrado que las especies del grupo $D$. onychophora, tienen preferencia de hospedero y están relacionadas a plantas de la familia Asteraceae (HUNTER, 1979, 1988, 1992). Drosophila verbesinae sp. nov. siempre fue capturada en las flores de Verbesina sodiroi y D. pappobolusae sp. nov. en las flores de Pappobolus imbaburensis plantas de la familia Asteraceae.

Drosophila verbesinae sp. nov. se asemeja a $D$. freilejoni Hunter, 1979; sin embargo, las comparaciones de la genitalia del macho y de la hembra demuestran que se trata de especies diferentes. Los machos de $D$. freilejoni tienen los cerci parcialmente unidos (VILELA \& Bächli, 1990) y en $D$. verbesinae sp. nov. los cerci son libres. Las hembras de $D$. freilejoni tienen oviscapto rectangular con aproximadamente 70 dientes y las hembras de $D$. verbesinae sp. nov. presentan oviscapto triangular con 100 a 140 dientes. Además las dos especies muestran preferencia hacia diferentes plantas hospederas. Drosophila freilejoni (Colombia) está asociada a Espeletia spp. (WINKER, 2005) y D. verbesinae sp. nov. a Verbesina sodiroi, por lo que no se consideran la misma especie, pero se sugiere que podrían ser especies gemelas.

Drosophila sisapamba sp. nov. y D. wayta sp. nov. probablemente pertenezcan al grupo $D$. onychophora ya que fueron capturadas en flores de asteráceas. Drosophila sisapamba sp. nov. presenta un edeago alargado y curvo similar a algunas especies del grupo $D$. onychophora. Por otro lado el edeago de $D$. wayta sp. nov. es similar a los edeagos de D. yurag sp. nov. y D. yuragshina sp. nov. (Figuero
\& RAfael, 2011), especies del grupo D. onychophora capturadas en el páramo de Papallacta en el 2009.

Drosophila arane es un nuevo registro para el Ecuador. La distribución de esta especie se amplía hasta Ecuador y hasta $2.900 \mathrm{~m}$ de altitud ya que el holotipo fue colectado en Colombia a 2.600 m (HunTER, 1992).

Agradecimientos. A la Pontifica Universidad Católica del Ecuador por financiar los proyectos no. E29050 y no. E29181. Al Ministerio de Ambiente por emitir la autorización de investigación científica no. 008-09 IC-FAU-DNB/MA. Al Dr. Carlos Ribeiro Vilela (Universidad de São Paulo), por sus valiosos comentarios sobre las nuevas especies del grupo D. onychophora. Al Dr. Guillermo Ayerve por su ayuda en la latinización de los nombres de las nuevas especies. A las licenciadas Gabriela Pozo y Fernanda Salazar y al personal del laboratorio de Genética Evolutiva de la PUCE. A Idea Wild por la donación de materiales.

\section{REFERENCIAS BIBLIOGRÁFICAS}

Bächli, G.; Vilela, C. R.; Escher, S. A. \& Saura, A. 2004. The Drosophilidae (Diptera) of Fennoscadia and Denmark. Fauna Entomologica Scandinavica 39. Leiden, Boston, Brill. 362p.

BRNCIC, D. 1966. Ecological and cytogenetical studies of Drosophila flavopilosa, a neotropical species living in Cestrum flowers. Evolution 20:16-29.

1983. Ecology of flower-breeding Drosophila. In: AsHBurner, M. A.; Carson, H. L. \& Thompson, J. N. eds. The genetics and biology of Drosophila. London, New York, Academic Press. v.3. p.333-382.

DAJoz, R.1999. Entomología Forestal: Los insectos y el bosque: papel y diversidad de los insectos en el medio forestal. Madrid Ediciones Mundi-Prensa. 550p.

dos Santos, R. C. O. \& Vilela, C. R. 2005. Breeding sites of Neotropical Drosophilidae (Diptera). IV. Living and fallen flowers of Sessea brasiliensis and Cestrum spp. (Solanaceae). Revista Brasileira de Entomologia 49:544-551.

Figuero, M. L. \& Rafael, V. 2011. Dos nuevas especies del grupo Drosophila onychophora (Diptera, Drosophilidae) en los bosques de Polylepis de Papallacta, Pichincha, Ecuador. Iheringia, Série Zoologia 101(4):343-349.

Hunter, A. S. 1979. New Anthophilic Drosophila of Colombia Annals of the Entomological Society of America 72:372-383. 1988. High Altitude Flower-Breeding Drosophila (Diptera: Drosophilidae). Pan-Pacific Entomologist 64(4):299-312.

1992. Flower-breeding Drosophila of Bogota, Colombia: New species (Diptera: Drosophilidae). Pan-Pacific Entomologist 68:192-199.

PIPKIn, S.; RodríguEZ, R. \& LEÓn, J. 1966. Plant host specificity among Flower-Feeding Neotropical Drosophila (Diptera: Drosophilidae). American Naturalist 100:135-156.

Silva, A. \& Martins, M. 2004. A new anthophilic species of Drosophila Fallén belonging to the bromeliae group of species (Diptera, Drosophilidae). Revista Brasileira de Zoologia 21:435-437.

Sturtevant, A.H. 1921. Genetic studies on Drosophila simulans. II. Genetics 6:43-64.

Val, F.; Vilela, C. \& Marques, M. 1981. Drosophilidae of the Neotropical region. In: Ashburner, M. A.; CARson, H. L. \& Thompson, J. N. eds. The genetics and biology of Drosophila. London, New York, Academic Press. v.3. p.123-168.

Vela, D. \& Rafael, V. 2004. Dos nuevas especies del grupo flavopilosa, género Drosophila (Diptera, Drosophilidae) en el Bosque Pasochoa. Revista Ecuatoriana de Medicina y Ciencias Biológicas 26:33-39.

Vilela, C. \& BÄChli, G. 1990. Taxonomic studies on Neotropical species of seven genera of Drosophilidae (Diptera). Bulletin de la Societé Entomologique Suisse 63(Suppl.):1-332

Winker, K. 2005. Sibling Species. The Auk 122:706-707. 